\title{
A simple way of approximating the canonical partition functions in statistical mechanics
}

\author{
Francisco M. Fernánded* \\ INIFTA (UNLP, CCT La Plata-CONICET), Blvd. 113 y 64 S/N, \\ Sucursal 4, Casilla de Correo 16, 1900 La Plata, Argentina
}

\begin{abstract}
We propose a simple pedagogical way of introducing the Euler-MacLaurin summation formula in an undergraduate course on statistical mechanics. We put forward two alternative routes: the first one is the simplest and yields the first two terms of the expansion. The second one is somewhat more elaborate and takes into account all the correction terms. We apply both to the calculation of the simplest one-particle canonical partition functions for the translational, vibrational and rotational degrees of freedom.

PACS numbers: 05.30.-d
\end{abstract}




\section{INTRODUCTION}

In statistical mechanics the thermodynamic functions are given in terms of the logarithm of the partition function and its derivatives with respect to volume, temperature, etc. The mathematical expression of the canonical partition function is an infinite sum over all the states of the system or, under some simplifying assumptions, the states of the individual particles. $\frac{1}{}$ Some of these infinite sums are commonly calculated approximately by means of the Euler-MacLaurin summation formula ${ }^{2}$ and most textbooks simply show how to apply it to the cases of interest. $\frac{1}{-}$ A rigorous derivation of the summation formula may be rather too demanding for inexperienced students and one avoids it in introductory courses on statistical thermodynamics or statistical mechanics.

There is a relatively simple way of deriving the summation formula by means of oper-

ator methods ${ }^{3}$ but it also requires some kind of mathematical expertise that students of introductory courses may not posses. However, the students may feel more confident and confortable if they are shown how to carry out such calculations by means of mathematical methods that they have already learned in a first course on mathematical analysis.

In an introductory undergraduate course we show how to obtain the first two terms of the Euler-MacLaurin summation formula in an extremely simple way that only requires the students to be familiar with the Taylor expansion. The aim of this paper is to put forward this approach that we deem suitable for pedagogical purposes. In section II we outline the method and apply it to some simple examples in section III. If we decide to show the students how to obtain terms of higher order this simple approach results to be rather cumbersome. For that reason, in section IV we show how to derive the full summation formula by a relatively minor change of strategy that requires some additional mathematical skills. The reader may choose one or another approach depending on the level of the course. Finally, in section $\mathrm{V}$ we summarize the results and draw conclusions.

\section{THE SIMPLEST APPROXIMATION}

The simplest canonical partition functions in statistical mechanics can be expressed as sums of the form

$$
S=\sum_{n=0}^{\infty} f(n)
$$


where $f(n)$ should tend to zero sufficiently fast when $n \rightarrow \infty$ because, otherwise, the sum does not converge. In most cases one cannot obtain this sum in closed form and therefore resorts to some kind of approximation valid under certain conditions; for example, sufficiently high temperature. In order to derive such an approach we define

$$
S(x)=\sum_{n=0}^{\infty} f(n+x),
$$

that satisfies

$$
\begin{aligned}
S(0) & =S \\
\lim _{x \rightarrow \infty} S(x) & =0,
\end{aligned}
$$

and

$$
S(x)-S(x+1)=f(x) .
$$

Substituting the Taylor expansion $S(x+h)=S(x)+S^{\prime}(x) h+\frac{1}{2} S^{\prime \prime}(x) h^{2}+\ldots$ for $h=1$ into (4)

we obtain, after some rearrangement,

$$
-S^{\prime}(x)=f(x)+\frac{1}{2} S^{\prime \prime}(x)+\ldots
$$

If we integrate this expression between $x$ and $\infty$ and take into account that $S(x)$ and all its derivatives vanish at the upper limit we have

$$
S(x)=\int_{x}^{\infty} f(t) d t-\frac{1}{2} S^{\prime}(x)+\ldots
$$

that can be solved iteratively. In the first step we omit the derivatives of $S(x)$ so that this function is approximately given by $S(x) \approx \int_{x}^{\infty} f(t) d t$. If we substitute this approximate result into the right-hand side of (6) we have

$$
S(x) \approx \int_{x}^{\infty} f(t) d t+\frac{1}{2} f(x) .
$$

Thus, for $x=0$ we obtain a simple approximation to the sum (11):

$$
S(0)=S \approx \int_{0}^{\infty} f(t) d t+\frac{1}{2} f(0)
$$

The simple method just outlined is suitable for introductory courses because it only requires basic knowlegde in mathematical analysis. This procedure is not suitable for the 
systematic calculation of the corrections of higher order to the basic formula (8) because it soon becomes rather cumbersome. However, this result is suitable for most porposes in an introductory course on statistical mechanics because several partitions functions for atomic and simple molecular systems can be easily derived from it. $\underline{\underline{1}}$

\section{SOME SIMPLE EXAMPLES}

Under some simplifying assumptions that we will not discuss in this paper statistical mechanics tell us how to express the thermodynamic functions of atomic and molecular systems in terms of one-particle partition functions of the form 1

$$
q(V, T)=\sum_{n} g_{n} e^{-\epsilon_{n} /\left(k_{B} T\right)}
$$

where $V$ is the volume of the container, $T$ the absolute temperature, $\epsilon_{n}$ the $n$-th energy level (assumed to be $g_{n}$-fold degenerate) of the particle and $k_{B}$ the Boltzman constant.

Let us first consider a particle of mass $m$ in a one-dimensional box of length $L$ with impenetrable walls which is the starting point for the calculation of the thermodynamic properties of an ideal monoatomic gas. ${ }^{1}$ If the particle has no internal structure its spectrum is only given by the translational degree of freedom:

$$
\epsilon_{n}^{t}=\frac{\hbar^{2} \pi^{2} n^{2}}{2 m L^{2}}, n=1,2, \ldots
$$

where $\hbar$ is the Planck constant $h$ divided $2 \pi$ and $g_{n}=1$. Upon defining $\alpha=h^{2} /\left(8 m k_{B} T L^{2}\right)$ the partition function $q_{t}$ reads

$$
\begin{aligned}
q_{t} & =S_{t}-1 \\
S_{t} & =\sum_{n=0}^{\infty} e^{-\alpha n^{2}}
\end{aligned}
$$

and the approximation (8) yields

$$
S_{t} \approx \frac{\sqrt{\pi}}{2 \sqrt{\alpha}}+\frac{1}{2}
$$

This approximate expression is accurate for sufficiently small values of $\alpha$ or, equivalently, when the thermal de Broglie wavelength $\Lambda=h / \sqrt{2 \pi m k_{B} T}$ is much smaller than the box length $L \underline{\underline{1}}$ The addition of corrections of higher order commonly improves the result of the approximate partition function, but in this case all of them vanish. $\frac{3}{}$ Figure 1 shows that this approximate expression becomes increasingly more accurate as $\alpha$ decreases. 
The spectrum of a one-dimensional harmonic oscillator is given by

$$
\epsilon_{n}^{v}=\left(n+\frac{1}{2}\right) h \nu, n=0,1, \ldots,
$$

where $\nu$ is the frequency of the oscillation and $g_{n}=1$. The partition function for this degree of freedom is given by

$$
\begin{aligned}
q_{v} & =e^{-\alpha / 2} S_{v}, \\
S_{v} & =\sum_{n=0}^{\infty} e^{-\alpha n}
\end{aligned}
$$

where $\alpha=h \nu /\left(k_{B} T\right)$. In this case the sum $S_{v}$ is a geometric series that can be calculated exactly and therefore we can derive its small- $\alpha$ expansion in a straightforward way:

$$
S_{v}=\frac{1}{1-e^{-\alpha}}=\frac{1}{\alpha}+\frac{1}{2}+\frac{\alpha}{12}-\frac{\alpha^{3}}{720}+\ldots
$$

If we define the vibrational temperature $\theta_{v}=h \nu / h_{B}$ then $\alpha=\theta_{v} / T$ and the EulerMacLaurin formula is a good approximation when $\theta_{v} \ll T$. The approximate expression (8) yields the first two terms of this series exactly.

The last example is the partition function for a rigid rotor with moment of inertia $I$. In this case, every energy level

$$
\epsilon_{J}^{r}=\frac{\hbar^{2}}{2 I} J(J+1), J=0,1, \ldots
$$

is $(2 J+1)$-fold degenerate and the partition function for the rotational degree of freedom is given by

$$
q_{r}=S_{r}=\sum_{J=0}^{\infty}(2 J+1) e^{-\alpha J(J+1)},
$$

where $\alpha=\hbar^{2} /\left(2 I k_{B} T\right)$. The approximate expression (8) yields

$$
S_{r} \approx \frac{1}{\alpha}+\frac{1}{2}
$$

that is accurate enough if $\alpha$ is sufficently small as shown in figure 2. It is custommary to define the rotational temperature $\theta_{r}=\hbar^{2} /\left(2 I k_{B}\right)^{1}$ so that $\alpha=\theta_{r} / T \ll 1$ when $\theta_{r} \ll T$. It is worth mentioning that the $\alpha$-independent term in equation (18) is not exact as shown in the following section. 


\section{SYSTEMATIC APPROACH}

If we decide to show the students how to obtain expression of higher order it is convenient to proceed in a different way. If we write the Taylor expansion discussed in section \I as

$$
S(x+1)=\sum_{j=0}^{\infty} \frac{1}{j !} S^{(j)}(x),
$$

where $S^{(j)}(x)$ is the $j$-th derivative of $S(x)$ with respect to $x$, then equation (6) reads

$$
S(x)=F(x)-\sum_{j=1}^{\infty} \frac{1}{(j+1) !} S^{(j)}(x),
$$

where $F(x)=\int_{x}^{\infty} f(t) d t$.

Instead of trying to solve equation (20) iteratively we propose a solution of the form

$$
S(x)=\sum_{k=0}^{\infty} a_{k} F^{(k)}(x),
$$

where $F^{(k)}(x)=-f^{(k-1)}(x)$. In order to obtain the coefficients $a_{k}$ we substitute (21) into (20) and compare the coefficients of $F^{(n)}(x)$ in the left- and right-hand sides; the result is

$$
\begin{aligned}
& a_{n}=-\sum_{j=1}^{n} \frac{a_{n-j}}{(j+1) !}, n=1,2, \ldots, \\
& a_{0}=1 .
\end{aligned}
$$

We thus have

$$
S(x)=F(x)-\sum_{k=0}^{\infty} a_{k+1} f^{(k)}(x),
$$

and

$$
S=\int_{0}^{\infty} f(t) d t-\sum_{k=0}^{\infty} a_{k+1} f^{(k)}(0) .
$$

Straightforward inspection of the first coefficients $a_{n}$

$$
\begin{aligned}
& a_{1}=-\frac{1}{2}, a_{2}=\frac{1}{12}, a_{3}=0, a_{4}=-\frac{1}{720}, a_{5}=0, a_{6}=\frac{1}{30240}, a_{7}=0 \\
& a_{8}=-\frac{1}{1209600}, a_{9}=0, a_{10}=\frac{1}{47900160}
\end{aligned}
$$

suggests that $a_{2 j+1}$ vanish for all $j>0$. In order to prove this conjecture we consider the function

$$
u(x)=\frac{x}{e^{x}-1}
$$


that satisfies

$$
u(x)-u(-x)=-x
$$

If we substitute the Taylor expansion

$$
u(x)=\sum_{j=0}^{\infty} u_{j} x^{j}
$$

into equation (27) we conclude that $u_{2 j+1}=0$ for all $j>0$. If we now expand $\left(e^{x}-1\right) u(x)=x$ in a Taylor series about $x=0$ and compare the coefficients of $x^{n}$ in the left- and right-hand sides of the resulting equation we obtain a recurrence relation for the coefficients $u_{n}$ that is identical with equation (22) for the coefficients $a_{n}$. We thus conclude that $u_{n}=a_{n}$ for all $n$. Therefore, equation (24) reduces to

$$
S=\int_{0}^{\infty} f(t) d t+\frac{1}{2} f(0)-\sum_{k=1}^{\infty} a_{2 k} f^{(2 k-1)}(0) .
$$

It is worth noting that the coefficients $a_{j}$ are related to the Brillouin numbers $B_{j} \stackrel{2}{\underline{2}}$ in the following way: $a_{j}=B_{j} / j$ !.

The operator method leads to this result in a more straightforward way and is also more convenient for the discussion of the radius of convergence of the series. $\frac{3}{3}$ However, we do not discuss it here because it requires the introduction of functions of operators that may not be suitable for an undergraduate course.

Let us apply this more accurate approach to the examples discussed in section [II] As pointed out in that section nothing can be done with the particle in a box because all the corrections of higher order vanish. This surprising result can be explained very easily and applies to all the sums in which $f(-x)=f(x)$ because $f^{(2 k-1)}(-x)=-f^{(2 k-1)}(x)$ and $f^{(2 k-1)}(0)=0$. A more rigorous analysis of such problems, based on the Poisson summation formula, can be found eslewhere. ${ }^{3}$

The harmonic oscillator is a suitable simple example for testing the summation formula (29) because we can calculate the exact expansion as shown in equation (15). In this case every new term added to the summation formula (29) yields one more term of the small- $\alpha$ series (15) as one may easily verify.

The application of the summation formula (29) to the partition function for the rigid rotor should be carried out with care. The reason is that $f^{(2 k+1)}(0)=\alpha^{k} P_{k+1}(\alpha)$, where $P_{k+1}(\alpha)$ is a polynomial function of $\alpha$ of degree $k+1$ and $P_{k+1}(0) \neq 0$. Therefore, the 
summation formula that includes all the terms through $f^{(2 k+1)}(0)$ only yields the small- $\alpha$ series correctly through degree $k$. For this reason the simple expression (8) does not yield the correct $\alpha$-independent term that receives contributions from $f(0)$ and $f^{\prime}(0)$. The expansion accurate to $\alpha^{4}$ is easily shown to be

$$
S_{r}=\frac{1}{\alpha}+\frac{1}{3}+\frac{\alpha}{15}+\frac{4 \alpha^{2}}{315}+\frac{\alpha^{3}}{315}+\frac{4 \alpha^{4}}{3465}+\ldots
$$

if we add all the terms trough $f^{(9)}(0)$ in the summation formula (29) as argued above. Since this series does not converge $\mathrm{e}^{3}$ one should truncate it before the terms start to increase.

\section{CONCLUSIONS}

As stated in the introduction we show the students the simple method developed in section II because it is sufficient for the purposes of our course. However, if a motivated student wants to learn how to obtain the corrections of greater order that appear in some of the available texbooks on statistical mechanics then one can suggest him or her to try the systematic approach of section IV or even the operator method. $\underline{3}$ In our opinion the present way of deriving the Euler-MacLaurin summation formula for statistical mechanics applications is more convenient for pedagogical purposes than the traditional one that appears in most textbooks on mathematics or numerical analysis. $\stackrel{4}{*}$ Such traditional approaches are certainly more rigorous and general but require the students to be more experienced in mathematics.

In passing, it is worth mentioning that the summation formula given by equations (22) and (29) is suitable for motivating the students to resort to a computer algebra system. In this way they bypass the tedious algebraic manipulation of the equations that is necessary for the calculation of contributions of large order like those in equation (30) and practise programming in any of such useful languages.

* Electronic address: fernande@quimica.unlp.edu.ar

1 D. A. McQuarrie, Statistical Mechanics, (Harper \& Row, New York, 1973).

2 M. Abramowitz and I. A. Stegun, Handbook of Mathematical Functions, Ninth ed. (Dover, New York, 1972). 
3 F. M. Fernández and E. A. Castro, Algebraic Methods in Quantum Chemistry and Physics, Mathematical Chemistry Series, (CRC, Boca Raton, New York, London, Tokyo, 1996).

4 T. M. Apostol, "An elementary view of Euler's summation formula", Amer. Math. Month. 106, 409-418 (1999). 


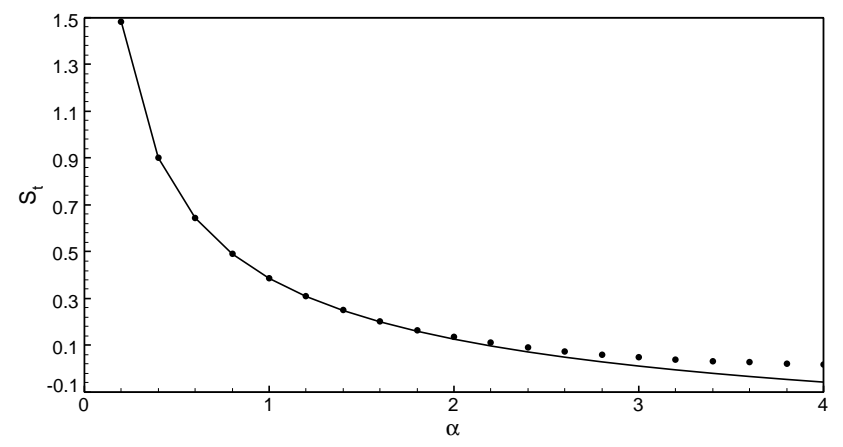

FIG. 1: $S_{t}$ calculated by means of the sum (11) (points) and the simple Euler-MacLaurin expression (12) (solid line)

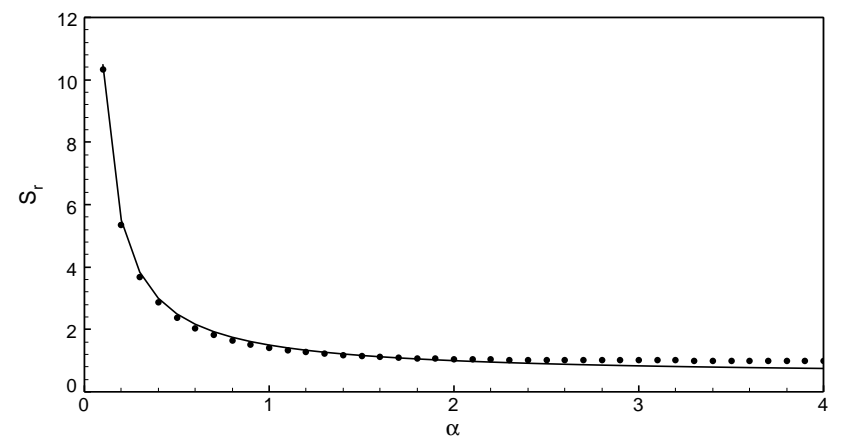

FIG. 2: $S_{r}$ calculated by means of the sum (17) (points) and the simple Euler-MacLaurin expression (18) (solid line) 